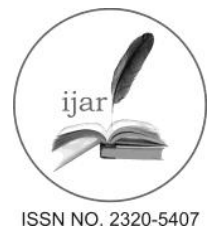

\section{Journal homepage: http://www.journalijar.com Journal DOI: $10.21474 / I J A R 01$}

RESEARCH ARTICLE

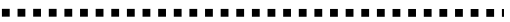
INTERNATIONAL JOURNAL OF ADVANCED RESEARCH

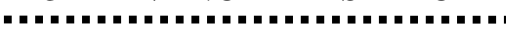

\title{
Study on FR Mechanism for User Privacy Protection on Social Networking Websites: An Overview.
}

Neha Anot and K.K. Singh.

Department of Electronics and Communication Engineering, Amity University, Lucknow.

\section{Manuscript Info}

\section{Manuscript History:}

Received: 15 March 2016

Final Accepted: 12 May 2016

Published Online: May 2016

Key words:

Cryptography, Fuzzy Neural

Network, Zernike Moments, SVM.

*Corresponding Author

..........................

Neha Anot.

\begin{abstract}
As the technology is progressing day by day, so is the numeral of safekeeping issues related to online social networking sites are growing. In this era, online social networks (OSNs) have prolonged a lot of fame around the globe. The OSN users share photos, videos and countless similar media, which at times are a big privacy issues for other users on OSN's. The privacy fears rise while certainusers update information of other users on social networking websites, which can be immaterial, or somebody wanted it to hide from others or kept it undisclosed. Such sort of information maycomprise of users images, his/her status updates, his/her videos, etc. The main objective of study of this paper is to deal with various kind techniques that can be used to solve the problem of safety related concerns of online social networks. The whole emphasis has been curiouslyplaced on the multiple entity-based images shared by the users, which comprise of privacy related issues. From the time when the decision on photo posting includesgroups in the circle (the most likely scenario) and it is dispersed in nature, our difficulty can be distorted to be a characteristic secure multiparty computation problem. Instinctively, we may ask cryptographic technique to protect privacy related issues, but the computational and communication cost may pose a severe problem for a large Online Social Networks. Apart from cryptographic approach, we may take the consensusbased approach as a promising alternative. The idea to let both user only deal with his/her local data and get the local training result, and then the neighboring users only need to exchange their training results. In the following round, each user still works on his own training data, but take the training results from his/her neighbors as references. We assume that the information will be spread over the OSN and everybody involved will extendthe same conclusion.
\end{abstract}

Copy Right, IJAR, 2016.. All rights reserved.

\section{Introduction:-}

Online Social Networks (OSNs) have ended up necessary piece of our day-by-day life and have greatly changed the way we connect with one another, satisfying our social needs-the requirements for social cooperation's, in context with todays phase of presentdevelopment sharing, gratefulness and appreciation. Online networking that makes individuals put more substance, including photographs, over OSNs without an excessive amount of thought on the substance. However, once something, for example, a photograph, is posted on the web, it will be recorded for all time, which might be utilized for any reasons. For instance, a posted photograph in a gathering might uncover an association of a big name to a mafia world. Because OSN clients might be negligent while posting content while the impact is as such achieving, security insurance over OSNs turns into an essential issue. Included more components, for example, photograph sharing and labeling are, the circumstance turns out to be more entangled. At the point when the informal organization clients endeavor to share a gathering photograph that contains people (photograph co-proprietors) other than himself/herself, for the present we are given any such arrangement by any of the 
interpersonal organization to confine from posting the gathering photographs. Despite what might be expected, informal community administration suppliers like Facebook, Instagram are rousing clients to post co-photographs and tag their companions out keeping in mind the end goal to get more individuals included. Just when the coproprietors will approve to share their data, the substance of the specific client will be indicated online and stay obscured something else. The unavoidable issue emerges with regards to the inquiry that what amount of control ought to be given to the co-proprietors to share the picture? To take care of the issue in this proposed model we are going to propose the uncommon protection imperative issue for the gathering photographs on the web. This model is gone for programmed labeling and character perception to ensure the client personality in the gathering photograph. By insights from Facebook, 95\% of the clients are labeled in any event once on a photograph, while another person labels generally photographs. Labeling uncovers personality quickly, however it likewise fills in as a notice. There are 350 million photographs transferred each day, thus imagine a scenario where somebody just transfers photographs containing a client without his/her insight. Presently, we can scarcely make a move. The anticipated gathering photograph security model will use the secured multi-party responsibility for gathering photograph. The cryptographic or obscure guide based method would be used to ensure the personality of the client who denied the solicitation by obscuring the face district of the particular client. Other than the character security model utilizing cryptography or obscure guide, the agreement-based methodology can be likewise used as the distinct option for accomplish the required errand. The proposed model will exhibit the proposed technique with higher genuine positive rate and false positive rate. Additionally the outcomes would be exhibited as review, exactness and precision.

\section{Brief literature survey:-}

J.Y. Choi, W. De Neve, K. Plataniotis, and Y.- M. Ro. (2011) has chipped away at face comment for powerful administration of individual photographs in online informal organizations (OSNs) is at present of extensive down to earth interest. Specifically, our communitarian FR system comprises of two noteworthy parts: choice of FR motors and combining (or combination) of various FR results. The choice of FR motors goes for deciding an arrangement of customized FR motors that are suitable for perceiving inquiry face pictures fitting in with a specific individual from the OSN. A. Besmer and H. Richter Lipford (2010) has dealt with Photo labeling. It is a well known component of numerous interpersonal organization destinations that permits clients to clarify transferred pictures with the individuals who are in them, unequivocally connecting the photograph to each per-child's profile. In this paper, they have inspected the security concerns and instruments encompassing these labeled pictures. P. A. Forero, A. Cano, and G. B. Giannakis (2010) has created calculations to prepare bolster vector machines when preparing information are conveyed crosswise over various hubs, and their correspondence to a concentrated handling unit is denied because of, for instance, correspondence unpredictability, adaptability, or security reasons. To perform this objective, the incorporated straight SVM issue is given a role as an arrangement of decentralized raised enhancement sub-issues (one for every hub) with accord limitations on the needed classifier parameters. K. Choi, H. Byun, and K. -A. Toh(2008) has taken a shot at Face acknowledgment has numerous helpful applications spreading over reconnaissance, law enforcement, data security, smart card and diversion innovations. Recently, a learning based face acknowledgment framework is likewise seen to be connected to web stage joining face acknowledgment and web administration. B. Carminati, E. Ferrari, and A. Perego(2006) has proposed Webbased interpersonal organizations (WBSNs) are online groups where members can set up connections and offer assets over the Web with different clients. Lately, a few WBSNs have been receiving Semantic Web innovations, for example, FOAF, for speaking to clients' information and connections, making it conceivable to authorize data exchange over different WBSNs. Regardless of its focal points as far as data dissemination, this raised the need of giving substance proprietors more control on the conveyance of their assets, which might be gotten to by a group far more extensive than they anticipate

\section{Conclusions of literature survey:-}

The choice on the gathering photograph model has been taken for the proposed model, which will secure the character of the clients in the circle of the client sharing the gathering photograph. The proposed model fits in with the multi-party computational issue. In this task, we propose a technique to help photograph's co-proprietors to get some control over their co-photographs. We keep up that the co-proprietor of a photograph must have the same control over the photograph as the proprietor. Regardless of whether to post the photograph ought to be an aggregate choice of everyone in the photograph. To accomplish the objective, the photograph-distributed action on OSNs will be effectively followed and checked and the gathering photograph protection model will be instated while a percentage of the client shares the gathering photograph over the OSN. At whatever point a client endeavors to post 
a photograph, he/she will get a notice and needs to settle on the joint choice on whether to post it or not with all people included in the photograph. In this way, a face acknowledgment motor (FR) is expected to perceive clients in the photograph. Photographs to be posted for the most part contain social companions on OSN, and subsequently, FR can be prepared to perceive the social companions (individuals in the social circle). Preparing procedures could be adjusted from the off-the-rack FR preparing calculations. FR with higher acknowledgment proportion requests all the more preparing tests (photographs of companions). However, online photograph assets may not contain enough photographs of a companion conceivably in a photograph. Clients who truly think about their photograph security are unrealistic to post an excess of photographs on the web. Maybe it is precisely those individuals who truly need to utilize our proposed component and expect a high acknowledgment proportion. To breakdown this issue, we offer a security protecting dispersed aggregate preparing framework for our FR with clients' private photographs as the preparation data.

\section{Face Detection and recognition model:-}

The current framework is utilizing the viola-jones calculation with the end goal of face location consolidated with the neural system with the end goal of the manufactured neural system (ANN). The current framework is putting forth the exactness of 87.05 percent which makes it less proficient for the live applications. Viola-jones is an exact and quick calculation with the end goal of face identification. Be that as it may, viola-jones is not productive regarding false positives. Additionally it needs learning in the beginning stages, which is unrealistic in the greater part of the times. Though the skin shading based face discovery can be demonstrated as more effective as far as precision, velocity and the false positives. The false positive are the outcomes got on the premise of measurable sort 1 and sort 2 mistakes. The false positive results speak to the recognition of wrong face identification.

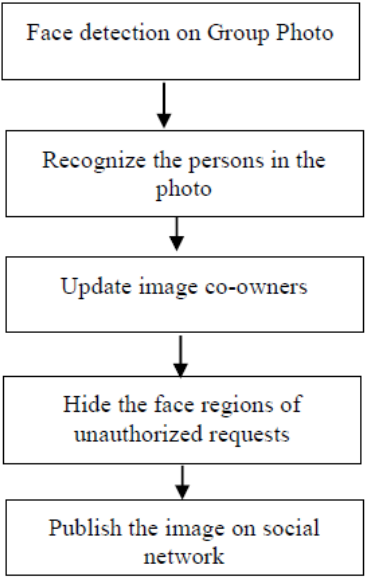

Figure 3: Working flowchart model of the proposed work

The neural system is likewise learning based bio-roused calculation, which is precise, hearty however moderate. The moderate pace of the neural system can be relieved utilizing the fluffy sets for the preprocessing of the face layouts and short-posting the most coordinating formats before running with the neural systems. With a pre-handling utilizing the fluffy sets will make the procedure speedier and productive than the current calculation. There is a solid need of some hearty element extractor for the face highlights with a specific end goal to enhance the precision of the face identification and acknowledgment model. For the exact face location and acknowledgment display, the Zernike minutes with SIFT can be utilized for the element extraction as a part of the ideal amalgamation. The Zernike minutes is the system used to check the position of the item, which can be utilized to recognize the face in the different edge development based minutes. The SIFT can be utilized for the imperative point extraction on the face locale, which can successfully check the contrast between the two face formats. In this examination, we reason an improving so as to compel and proficient Face discovery method existing face location calculations. This face location calculation must be successful, precise and productive. We are putting forth a novel and vigorous skin shading model and fluffy neural system based face identification with the end goal of validation where the framework will distinguish and perceive the persons utilizing the face pictures. We are putting forth a novel and hearty skin shading model and fluffy neural system based face recognition with the end goal of confirmation where the framework will identify and perceive the persons utilizing the face pictures. Face identification with the end goal of confirmation where the framework will distinguish and perceive the persons utilizing the face pictures. The new 
calculation will versatile to different face discovery based validation frameworks. This calculation would be planned utilizing mixes of skin shading model with fluffy neural system. The proposed model will be produced utilizing the skin shading model over Zernike minutes and SIFT for face location and highlight portrayal alongside neural system over insatiable for the face acknowledgment, where ravenous will be utilized to choose the right result in the tied circumstance in the middle of SIFT and Zernike minutes.

\section{Procedure:-}

Running in the setup mode, the project is working towards the foundation of the enhanced choice tree. For this reason, the private preparing set and the area should be determined. At the point when "Private preparing set" catch is squeezed, photographs in the advanced mobile phone displays could be chosen. To setup the area, at this stage, a client needs to physically select their Facebook companions with the catch "Pick companions". Notice that, all the chose companions are required to introduce our application to do the collective preparing. With Private preparing set and neighborhood determined, the setup mode could be initiated by squeezing the catch "Begin". Amid the preparation handle, an attachment is built up between any two clients to empower the correspondence. Through the attachments, Algorithm is performed to acquire the classifiers. After the classifiers are acquired, an enhanced choice tree is developed and the project changes from the setup mode to the resting mode. The system could be summoned to the working mode with the catch "Post Photo". Working in this mode, every one of the countenances on the posting photograph are distinguished and perceived, then, demands will send to the recognized companions. The solicitations will be appeared as a warning in their App Center Requests. Alongside the solicitation, the photograph will be sent to them. After accepting the post consents from the distinguished companions, the photograph is posted; something else, the posting activity will be lapsed in 2 weeks.

\section{Conclusion:-}

The proposed model is gone for using so as to comprehend the gathering photograph issue the face identification and client approval model. The novel face locale recognition calculation would be used with the end goal of individual recognizable proof present in the picture. The proposed model offers the dynamic associate based system model to approve the clients present in the given gathering photograph. The normal result is to accomplish the higher exactness with higher accuracy in the general consequences of the working model from the improvement of this model. The outcomes would got as exactness, accuracy and review parameters.

\section{Acknowledgement:-}

The authors are thankful to Hon'able C - VI, Mr. Aseem Chauhan (Additional President, RBEF and Chancellor AUR, Jaipur), Honáble Pro VC Maj. General K. K. Ohri (AVSM, Retd.) Amity University, Lucknow, Wg. Cdr. (Retd.) Dr. Anil Kumar, (Director, ASET), Prof. S. T. H. Abidi (Professor Emeritus), Brig. U. K. Chopra, Retd. (Director AIIT), Prof. H K Dwivedi (Director, ASAP), Prof O. P. Singh (HOD, Electrical \& Electronics Engg.) and Prof. N. Ram (Dy. Director ASET) for their motivation, kind cooperation, and suggestions.

\section{Refrences:-}

1. A. Besmer and H. Richter Lipford. Moving beyond untagging: photo privacy in a tagged world. In Proceedings of the SIGCHIConference on Human Factors in Computing Systems, CHI '10,pages 1563-1572, New York, NY, USA, 2010. ACM.

2. B. Carminati, E. Ferrari, and A. Perego. Rule-based access control for social networks. In R. Meersman, Z. Tari, and P. Herrero, editors, On the Move to Meaningful Internet Systems 2006: OTM2006 Workshops, volume 4278 of Lecture Notes in Computer Science, pages 1734-1744. Springer Berlin Heidelberg, 2006.

3. K. Choi, H. Byun, and K.-A. Toh. A collaborative face recognition framework on a social network platform.InAutomatic Face GestureRecognition, 2008.FG '08. 8th IEEE International Conference on,pages 16, 2008.

4. P. A. Forero, A. Cano, and G. B. Giannakis. Consensus-based distributed support vector machines. J. Mach. Learn. Res., 99:1663-1707, August 2010.

5. J. Y. Choi, W. De Neve, K. Plataniotis, and Y.-M. Ro. Collaborative face recognition for improved face annotation in personal photo collections shared on online social networks. Multimedia, IEEETransactions on, 13(1):14-28, 2011. 
6. K. Choi, H. Byun, and K.-A. Toh. A collaborative face recognition framework on a social network platform.InAutomatic Face GestureRecognition, 2008.FG '08. 8th IEEE International Conference on,pages 16, 2008.

7. K.-B. Duan and S. S. Keerthi. Which is the best multiclass svm method? An empirical study. In Proceedings of the 6th internationalconference on Multiple Classifier Systems, MCS'05, pages 278-285, Berlin, Heidelberg, 2005. Springer-Verlag

8. P. A. Forero, A. Cano, and G. B. Giannakis. Consensus-based distributed support vector machines. J. Mach. Learn. Res., 99:1663-1707, August 2010.

9. B. Goethals, S. Laur, H. Lipmaa, and T. Mielik?inen. On private scalar product computation for privacypreserving data mining.Proceedings of the 7th Annual International Conference in Information Security and Cryptology, pages 104-120. Springer-Verlag, 2004.

10. L. Kissner and D. Song. Privacy-preserving set operations. In INADVANCES IN CRYPTOLOGY - CRYPTO 2005, LNCS, pages241-257. Springer, 2005.

11. J. Hochreiter, et al., Exploring album structure for face recognition in online social networks, Image Vis. Comput. (2014). 